\title{
La industria de la confección zuliana en la era de la competitividad
}

\author{
Romero de Cuba, Jenny ${ }^{1}$ \\ Sandrea, Maryana ${ }^{2}$ \\ Morales, Mariher ${ }^{3}$ \\ Boscán, Mariby 4 \\ Acosta, Ana ${ }^{5}$
}

\section{Resumen}

La Industria de la confección constituye una de las actividades económicas de mayor dinamismo en el mercado internacional. Por esta razón, los gobiernos de algunos países, especialmente los de Asia han utilizado este sector como apoyo de sus procesos de industrialización, debido fundamentalmente al poder empleador que este presenta. Partiendo de la idea antes esbozada, el presente artículo se propone como objetivo analizar la situación actual que enfrenta esta industria en la región zuliana-Venezuela durante el periodo 1989-1995. Como fuente principal de información se utiliza la Encuesta Industrial elaborada por la Oficina Central de Estadística e Informática (OCEI). Los resultados revelan que en el periodo de estudio el sector se ha visto notablemente afectado por las medidas de ajuste estructural y la apertura de la economía. Particularmente, esta última ha traído como consecuencia una reducción de los niveles de producción y empleo en la región, producto de la persistente sobrevaluación del bolivar que ha permitido la entrada de una

\section{Recibido: $99-11-11$. Aceptado: 00-05-10}

1 Magister en Gerencia de Empresas. Investigadora del instituto de Investigaciones de la Facultad de Ciencias Económicas y Sociales (FCES) de la Universidad del Zulia (LUZ). Autor para la correspondencia. Telf. (Fax). 061-596513. E-mail: jennyrc@ hotmail.com.

2 Economista. Becaria Académica del Instituto de Investigaciones de la FCES-LUZ. Cursante de la Maestría en Gerencia de Empresas. Postgrado de Economia. FCES-LUZ.

3 Economista. Becaria Académica del Instituto de Investigaciones de la FCES-LUZ. Cursante de la Maestría en Gerencia de Empresas. Postgrado de Economia. FCES-LUZ.

4 Economista. Becaria Académica del Instituto de Investigaciones de la FCES-LUZ. Cursante de la Maestria en Gerencia de Empresas. Postgrado de Economia. FCES-LUZ.

5 Magister en Políticas Públicas. Investigadora del Instituto de investigaciones de la Facultad de Ciencias Económicas y Sociales de la Universidad del Zulia. 
gran cantidad de productos importados a precios notablemente inferiores, lo que aunado a la reducción de la capacidad de compra de la población determinan una contracción en el parque industrial. No obstante, a pesar de este panorama, existen en un futuro cercano posibilidades de desarrollo de dicho sector, en vista, de que el deterioro salarial al cual se ha visto sometido el venezolano ha abaratado notablemente el costo de la mano de obra, cuestión fundamental para el desarrollo de esta industria a partir del aprovechamiento de ventajas competitivas estáticas.

Palabras clave: Industria de la Confección, Región Zuliana-Venezuela, deterioro salarial, empleo, ventajas competitivas estáticas, pequeña industria.

\section{Dressmaking Industry in Zulia in the Competitive Era}

\section{Abstract}

The dressmaking industry constitutes one of the most dynamic economic activities in the international market. For this reason, the governments of certain countries, especially in Asian, utilize this sector as support for their industrialization process principally due to the employment possibilities it represents. Based on this understanding, this article proposes an objective analysis of the present situation that this industry faces in the Zulia region of Venezuela, based on an analysis of the period 1989-1995. As a principal source of information, the Industrial Census elaborated by the Central Office of Statistical Information (OCEI) was used. The results reveal that in the period under study, the dressmaking sector has been visibly affected by governmental structural adjustment policies, and by the opening of the economy to world markets. This last factor in particular has resulted in a reduction of production and employment levels in the region, due to the persistent over-evaluation of the Bolivar. This has permitted the entry of a large quantity of imported products at much lower prices, and which, when added to the lowered purchasing power of Venezuelans, has brought about a contraction in the industrial sector. However, in spite of this negative panorama, there is a promising future of development possibilities for this sector, since the salary deterioration which has affected Venezuela has notably lowered labor costs, which is a fundamental element in the development of this industry based on static comparative advantages.

Key words: Dressmaking industry, Zulia Region of Venezuela, salary deterioration, employment, static comparative advantages, small industry. 


\section{Introducción}

La industria de la confección funciona como una actividad globalizada en el mercado mundial. Ella ha constituido una pieza importante en el desarrollo de muchos paises (Rosales, 1994:1); de hecho algunas economías exitosas de reciente industrialización como las asiáticas (Corea, Taiwán, entre otras.) han iniciado sus procesos de modernización bajo la producción y fomento de este sector; cuya explotación les ha permitido aprovechar una de sus fuertes ventajas comparativas como es la baja tasa de salario existente; siendo este aspecto potenciado por sólidas ventajas competitivas orientadas hacia procesos productivos más eficientes, la introducción de nuevas tecnologías y una elevada tendencia hacia la diferenciación del producto.

A pesar de ser ésta una actividad con alto grado de competitividad internacional, por razones de tipo estratégico la mayoría de los países, bien sea industrializados o en desarrollo, han optado por fortalecer estas empresas debido a que las mismas responden a gustos y cos- tumbres muy particulares de sus pobladores (Rosales, 1994:1).

Venezuela no ha constituido la excepción de esta regla; prueba de ello es que durante más de treinta años dicho sector fue amparado por las políticas de sustitución de importaciones, las cuales favorecieron no sólo a la industria de la confección sino también al sector textil, debido al alto grado de interrelación entre ambos, que los lleva a ser considerados como una sola cadena productiva denominada cadena textil-confección. Es importante resaltar que concretamente en Venezuela la industria de la confección comprende las actividades de manufactura de prendas de vestir mediante el corte y costura de telas, cueros $y$ otros materiales ${ }^{1}$.

Por otra parte, el excesivo proteccionismo al cual fue sometida dicha cadena productiva fomentó la ineficiencia y la baja productividad que la caracteriza actualmente, esta situación dio lugar a un aumento indiscriminado de las importaciones en el sector confección, que aunado a la persistente sobrevaluación del bolivar y al aumento de la competencia des-

La Oficina Central de Estadísticas e Informática Venezolana (OCE) definió las actividades que agrupa el sector confección según la clasificación Industrial Internacional Uniforme (C.I.I.U.) de las Naciones Unidas (código 322); de acuerdo con la mencionada clasificación el sector confección comprende además las actividades de; confección de formas para sombreros, sombreros de todas clases, adomos y accesorios. Destacando entre los principales productos de este sector los trajes, ropa interior y de vestir; sombreros en general, prendas y accesorios de piel; guantes y mitones; tirantes, ligas y productos conexos; "robes de chambre" y batas, impermeables y otras prendas de vestir impermeabilizadas; ropa de cuero; ropa forrada en piel de oveja; cinturones de fantasia de cualquier material; pañuelos, birretes y togas académicas; hábitos sacerdotales, $y$ trajes para representaciones teatrales. 
leal, junto con los ilicitos aduaneros (contrabando) terminaron por colapsarlo.

La llegada de la política de apertura comercial propinó un duro golpe a esta industria, sumiéndola en un agudo retroceso de sus principales indicadores, expresados en términos del producto, empleo, salarios y número de establecimientos.

Si bien es cierto que el panorama descrito anteriormente configura un escenario negativo para el futuro de esta actividad, también lo es, que la expansión de dicha industria se orienta hacia la búsqueda de mano de obra abundante y barata. En Venezuela, particularmente en el estado Zulia, este aspecto, constituye un polo de atracción, dado el creciente deterioro del salario real, las altas tasa de desempleo y el elevado sector informal. A pesar de ser este argumento fundamental para la competitividad de la industria de la confección, no es el único factor a considerar; la experiencia en otros países sugiere que la capacidad gerencial, de innovación y de respuesta de las empresas son elementos cruciales para ingresar exitosamente en el mercado internacional.

\section{Evolución mundial de la industria de la confección}

Hasta 1960 la hegemonía en el complejo textil - confección correspondía a los países desarrollados, entre ellos Estados Unidos, Canadá, Francia e Italia (Rosales, 1994: 20). Pero debido, entre otras razones, al aumento en los costos de la mano de obra estos países se preocuparon por desarrollar industrias intensi- vas en tecnología, como por ejemplo: la industria de la informática, telecomunicaciones, biotecnología, entre otras, lo cual produjo cambios en la estructura del comercio mundial. Estos cambios provocaron modificaciones en la rama textil - confección, trasladándose ésta a los países asiáticos, donde experimenta un gran avance producto del establecimiento de políticas de industrialización basadas en el uso intensivo de mano de obra.

Cabe destacar que Estados Unidos desempeña un papel protagónico en el comercio internacional de textiles y prendas de vestir, siendo el primer importador mundial y uno de los más grandes exportadores de los rubros en cuestión (Molina y Alonso, 1991:1).

Adicionalmente, se debe mencionar que en los últimos diez años, el comercio de textiles y vestidos entre Estados Unidos y los países de América Latina ha aumentado considerablemente a la vez que otros grandes abastecedores han estado perdiendo posiciones durante la primera mitad de los años noventa, con lo cual se ha comenzado a desplazar a algunos países asiáticos.

Al respecto, Valentine Kouzmine (1997:4) expresa que las importaciones de textiles y prendas de vestir procedentes de América Latina han crecido a tasas elevadas y cobran cada vez mayor participación en el total de las importaciones estadounidenses $(57,7 \%$ para 1995 mientras que para 1990 era de $34,8 \%$ lo que representa un aumento del $22,9 \%$ ). Entre los factores que ejercen influencia sobre el auge exportador de los paises de América Latina y el Caribe destacan: 
a. Establecimiento de varios acuerdos y disposiciones legales firmados en los últimos años que le otorgan a los paises caribeños preferencias a través de las cuotas de importación exoneradas de impuesto.

El tratado de Libre Comercio de América del Norte (TLC), ha otorgado grandes ventajas a México (reducción de aranceles y eliminación inmediata de cuotas) para acceder al mercado estadounidense. En virtud de este convenio, se aplican las llamadas reglas de origen que suponen la utilización de materias primas procedentes de los países del TLC para que las prendas confeccionadas con ellas sean importadas hacia Estados Unidos con las facilidades mencionadas.

Otros tratados han permitido a países como Colombia y Perú, aumentar las exportaciones de la cadena textilconfección hacia el mercado estadounidense en los primeros cinco años de la década de los noventa, mientras que Brasil, Argentina y Uruguay han perdido participación en dicho mercado.

b. El sistema de subcontratación y de producción compartida (Maquila).

A través de este último mecanismo, las compañías norteamericanas pueden exportar a ciertos paises productos textiles en vias de conversión en prendas de vestir e importarlos luego que se confeccionen, pagando solamente el impuesto por el valor agregado. Países como Jamaica, Costa Rica, República Dominicana, Haití, entre otros, se han convertido recientemente en proveedores de mano de obra barata para el sector confección (Rosales, 1994: 23).

c. La denominada ventaja de la proximidad, sustentada en los menores costos de transportes y comunicación a causa de la relativa cercanía geográfica, ha impulsado el auge exportador de los países latinoamericanos y de algunas islas del caribe durante la década de los noventa, potenciando el establecimiento de acuerdos regionales firmados recientemente con los Estados Unidos.

En este sentido, destaca la industria textil-confección mexicana la cual es sumamente grande y goza de acuerdos preferenciales importantes con los Estados Unidos, así como de la ventaja de la proximidad en un mayor grado que el resto de los países de la región (Kouzmine, 1997: 16).

d. Otro aspecto a considerar en la amplitud del comercio con los Estados Unidos, está referido al tamaño de la Industria de la confección en los países latinoamericanos, donde resultan favorecidos aquellos que posean una mayor capacidad instalada, que permita satisfacer el incremento de la demanda derivada de los acuerdos comerciales.

Entre los paises de América Latina con mayor capacidad exportadora se encuentran: Colombia, México, Brasil, y Chile. La industria colombiana tiene una gran capacidad de generar excedentes para la exportación ya que cuenta con una tradición que se remonta a más de treinta años. En los años sesenta la industria textil colombiana era considerada la más desarrollada de la región andina e incluso 
de Latinoamérica, pero al sucumbir al facilismo de la protección perdió un importante nivel de competitividad internacional lo cual la ha hecho vulnerable ante las importaciones legales y el contrabando (Rosales, 1994: 25). La industria brasileña posee una larga y activa experiencia exportadora y un mercado interno bastante grande debido a la gran población existente en ese país. Por su parte la industria chilena ha logrado importantes acuerdos preferenciales para acceder al mercado norteamericano en virtud de su política de apertura económica; sin embargo, su participación ha sido modesta en los últimos años (Kouzmine, 1997: 16).

e. Uno de los factores que ha tenido mayor incidencia en el crecimiento reciente de las exportaciones latinoamericanas está relacionado con el bajo costo laboral.

Cabe destacar, que en épocas anteriores los países asiáticos liderizaban el rubro de exportaciones sustentán. dose en el bajo costo de la mano de obra, pero a medida que ha ido aumentando la productividad en estos paises se registró una notable mejoría en el salario de los trabajadores. Por el contrario, en el caso de los países de América Latina las crisis en el orden económico, político y social que a lo largo de los últimos años han venido experimentando, provocaron un sostenido deterioro salarial disminuyendo asi la calidad de vida de los trabajadores.

Ciertamente, el comercio de textiles y prendas de vestir entre Estados Unidos y Latinoamérica está creciendo a pasos agigantados, pero, sólo ciertos países de la región se benefician de ello, a pesar de que el tamaño del mercado permitiría la participación de otros países.

Venezuela podría incursionar en el mercado internacional de la industria de la confección, pues si se considera como parámetro para medir la competitividad en dicho sector los bajos costos salariales, este país presenta para 1997 en comparación con el año 1992, un deterioro importante en el índice de salario minimo rural urbano, al pasar de la posición No. 16 a la No. 6 , aspecto que lo coloca frente a algunos paises de América Latina y del Caribe como un fuerte competidor en industrias intensivas en mano de obra. Al respecto la Comisión Económica para América Latina y el Caribe (CEPAL) revela en su informe anual de 1997, una caída sostenida del índice promedio anual del salario minimo venezolano a partir de 1994. (Ver Tabla 1).

\section{Evolución de la industria de la confección venezolana}

Con el proceso de colonización se crearon nuevas y numerosas necesidades en relación al uso de prendas de vestir, hecho que propició la instalación de telares rudimentarios que permitieran la transformación de la materia prima (algodón recolectado por los indígenas) en productos confeccionados. Posteriormente, la producción textil y la confección del vestido se intensificaron con el objeto de satisfacer no sólo necesidades locales sino también el intercambio con otros paí- 


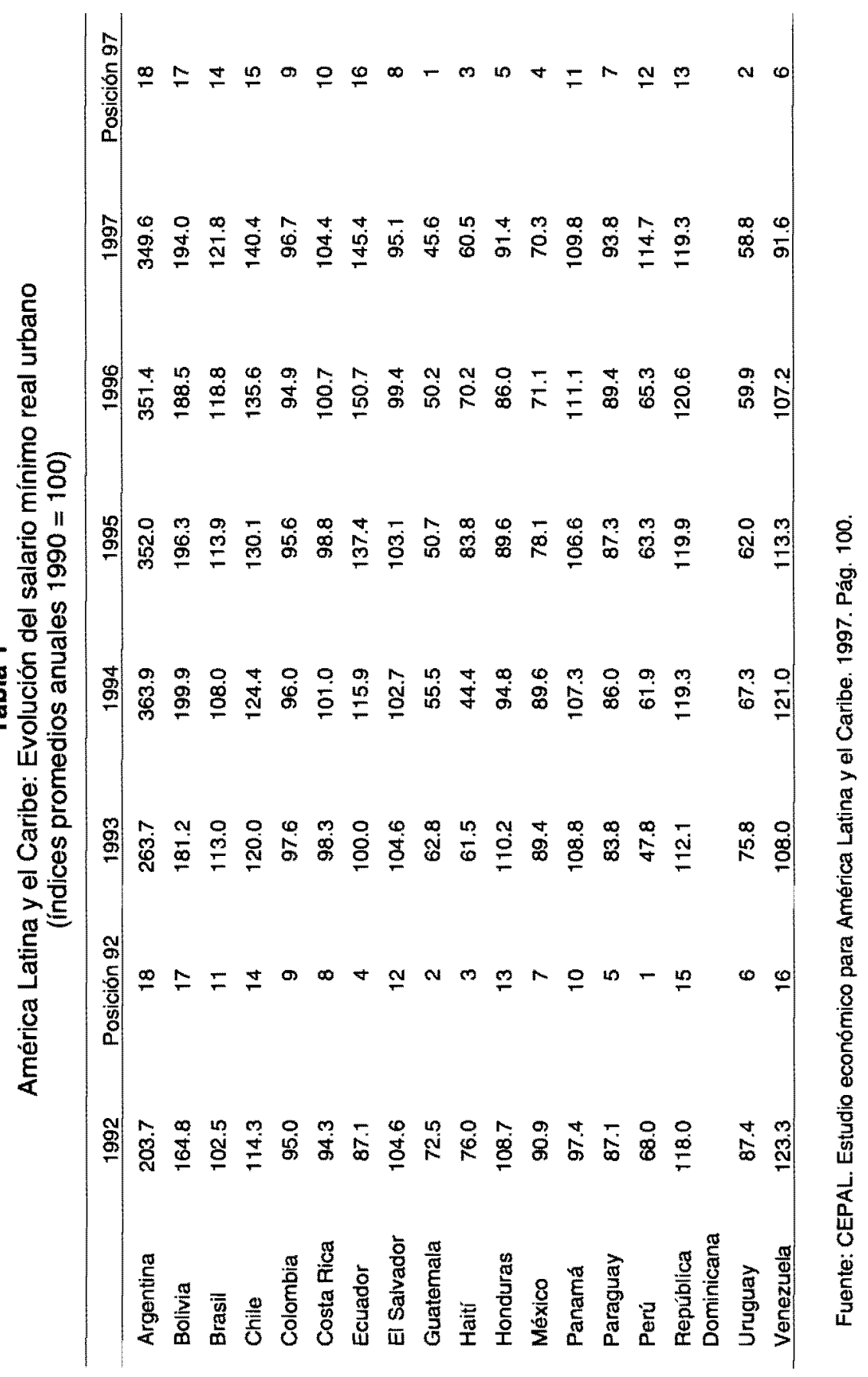


ses del Caribe. Para esa época, surgen industrias de mayor rentabilidad en el país como la del tabaco, el café y el cacao, por lo que se le resta importancia a la manufactura textilera naciente.

En la última parte del siglo $X I X$ comienzan a instalarse los primeros telares mecánicos para la producción de pabilo. A partir de 1914 surge en Venezuela la industria petrolera que por los cuantiosos recursos que genera provocó modificaciones en la estructura económica del pais, desplazando a otros sectores como la agricultura y la artesania, dentro de la cual destaca la industria textilera. Posteriormente, para 1930 se registra un crecimiento notable del sector textilero-confección; no obstante, cabe destacar que el mismo no estuvo acompañado por una tecnificación de la industria.

Con el advenimiento del modelo de sustitución de importaciones en la década de los años cincuenta se fortaleció el desarrollo del eje textil-confección, dado que durante la primera fase del mencionado proceso se le dio prioridad a la sustitución de importaciones de bienes de consumo final. Es de hacer notar, que otro de los factores que incidió en el crecimiento de este sector está relacionado con el aumento de la demanda de prendas de vestir por parte de los residentes nacionales, derivada de la expansión del poder adquisitivo que trajo consigo las exportaciones petroleras y el desarrolio de la vida urbana.

Todo lo antes expuesto trajo como resultado que para 1954 se iniciara un proceso de modernización de la industria textil, sustentado en una serie de políticas gubernamentales entre las que cabe destacar: aumento de los impuestos de im- portación, eliminación de licencias de importación, apoyo financiero para la adquisición de maquinarias y equipos que incorporaron los últimos adelantos tecnológicos a tasas de interés preferencial. Además de un escenario económico donde había gran disponibilidad de divisas y estabilidad de la tasa de cambio, hechos que favorecen al sector confección pues ayudan a crear materia prima local. El proceso de modernización mencionado anteriormente se evidencia al observar que de 1950 a 1958 el producto de la industria del vestido aumenta de $2,4 \%$ al $4,3 \%$ según cifras del B.C.V. (Bitar y Troncoso, 1983: 259).

Según Toledano y Castillo (1997), las medidas proteccionistas estuvieron vigentes hasta finales de los años sesenta lo que en cierta forma estimuló la dinamización del sector confección a nivel nacional; sin embargo, en el ámbito internacional esta industria se desempeñó de forma poco competitiva $e$ ineficiente. A partir de 1975 y hasta 1978 se observa un crecimiento y expansión en la industria de la confección, debido al proteccionismo que existía para las industrias tradicionales, lo cual propició el incremento en el número de establecimientos y en el personal ocupado, en especial en el sector de la pequeña y la mediana industria.

La etapa subsiguiente, que corresponde a la década de los ochenta, evidencia el inicio de la crisis en el sector confección, producto del derrumbe de las barreras arancelarias, que junto a la sobrevaluación del bolívar originó el aumento de las importaciones de este rubro.

Por otra parte, las condiciones de precio y calidad del producto que ofrecían 
las empresas del mercado interno estaban por debajo de los estándares internacionales, cuestión que demostró que las políticas proteccionistas, lejos de impulsar la competitividad fomentaron la ineficiencia en este tipo de empresas. Posteriormente, con las medidas adoptadas en febrero de 1983, se restableció el mercado nacional, dado que las mismas encarecieron las importaciones estimulando nuevamente la producción en el sector. Estas condiciones incentivaron el establecimiento de nuevas empresas y la renovación del parque industrial en el sector confección, iniciada con la nueva política de Reconversión Industrial.

\section{El sector de la confección venezolano en el marco de la competitividad}

La industria de la confección se encuentra inmersa dentro del proceso de globalización iniciado a partir de las últimas décadas del siglo $\mathrm{XX}$, destacando entre las caracteristicas esenciales del desarrollo de este sector, la dependencia de los tratados y acuerdos internacionales que regulan el comercio de productos confeccionados. Este rasgo de interconexión de mercados le imprime condicionamientos tanto a la producción como al comercio y al mismo tiempo implica que la supervivencia de industrias locales está ligada a la capacidad para insertarse en economias globales altamente competitivas.

En este sentido, es importante aclarar que la globalización está determinada por procesos concretos y simultáneos, destacando: 1) Una apertura de las fronteras nacionales a los flujos de co- mercio, servicios, inversiones, tecnología, innovaciones que se originan en diferentes partes del mundo. 2) La dinámica de la Competitividad, cuya dimensión no sólo trasciende a un aspecto microeconómico, es decir, a escala de empresas, sino a la macrocompetividad (a escala de país), cuyas ventajas competitivas correspondan al estado-nación como unidad macroeconómica o macropolítica. competitividad (Maza Zavala, 1999: 11).

Estas ventajas competitivas han permitido el desarrollo del comercio internacional, pues el mismo se constituye en un factor complementario de las economías nacionales, dado que los paises pueden dedicarse a lo que pueden producir mejor para lograr altos niveles de eficiencia y competir ventajosamente con algunos productos en el mercado mundial (Corona, 1996: 912).

Debido a la interdependencia mundial que se ha venido gestando, las ventajas comparativas se han ligado estratégicamente con la competitividad de las economias nacionales. Las ventajas competitivas, representan hoy una evolución de las ventajas comparativas, en su acepción general de indole estática, pero con la distinción fundamental y prioritaria de la época que viene determinada por la aptitud hacia el conocimiento, la calidad de gestión, la innovación en procedimientos de producción y comercialización, así como de organización y administración, de manejo de recursos tecnológicos superiores, experiencia en los negocios, entre otros.

No cabe duda, que el nuevo modelo de competencia internacional se orienta al desarrollo de las industrias sustentadas en las denominadas ventajas compe- 
titivas dinámicas, caracterizadas por la innovación tecnológica, la sostenibilidad ambiental, microelectrónica, telemática y la formación de recursos humanos. Sin embargo, existen otras empresas donde el factor fundamental para su impulso lo constituye otra dimensión de las ventajas competitivas relacionada con la dotación de recursos naturales, los costos de los factores de la producción (tierra, mano de obra, capital), la proximidad de los mercados de exportación, el clima y el acceso a diferentes tecnologías, conocidas como ventajas competitivas estáticas. (Corona, 1996: 912).

La Industria de la Confección constituye un ejemplo de éstas últimas, pues su expansión se ha dirigido básicamente hacia aquellos paises que presentan menores costos de mano de obra, motivo por el cual ha sido utilizada como instrumento para promover el desarrollo industrial en algunas naciones asiáticas como Corea, China y Taiwán e incluso latinoamericanas como México, Colombia y República Dominicana, debido a que es intensiva en mano de obra.

Los paises de América Latina recientemente han incursionado con éxito en el mercado textil-confección logrando desplazar incluso a algunos competidores asiáticos como proveedores de la industria norteamericana. Tal situación obedece a las condiciones económicas y sociales bajo las cuales labora la población en dicha región, que la convierten en uno de los proveedores más barato de mano de obra en confección. (Kouzmine, 1997: 7).

A pesar del panorama anteriormente descrito, la industría de la confección venezolana se enfrenta en la actualidad a serias distorsiones, entre las cua- les destaca, según Chiappe (1999), la sobrevaluación del bolívar, la alta competencia que en ocasiones puede ser "desleal", la falsificación de marcas, las altas tasas de interés y la inflación, lo cual ha provocado que a nivel nacional hayan cerrado alrededor de 320 industrias textiles de un total de 400 , en un lapso no mayor de cinco años.

Según la Cámara Venezolana de Industriales del Vestido (Citada por Chiappe, 1999), las exportaciones venezolanas en estos rubros han caído progresivamente en los últimos tres años, debido a que después de vender entre 41 y 48 millones de dólares anuales entre 1993 y 1996, el volumen de ingresos por exportaciones cayó a 31 millones en 1997 y 18.5 millones en 1998.

Este comportamiento negativo en las exportaciones es atribuido a una politica monetaria nacional restrictiva diseñada para combatir las alta tasa de inflación combinada con una política cambiaria que mantiene a decir de los voceros de la Cámara de Industriales del Vestido sobrevaluado el signo monetario nacional, lo cual encarece los productos venezolanos frente a los de la competencia extranjera.

Por otra parte, el cierre de las industrias ha llevado consigo una caida en el empleo del $59.22 \%$, mientras que las empresas del sector formal se están pasando al sector informal, como vía para reducir los costos, o bien se han cambiado de rama, es decir, de fabricantes a simples distribuidores, entrando entonces al sector comercio en busca de una mayor rentabilidad.

La sobrevaluación del bolívar, por su parte, torna poco competitivos los productos venezolanos, debido a los altos 
precios que presentan frente a sus competidores del exterior. Los productores de prendas de vestir nacionales se sienten desprotegidos pues, mientras en otros países andinos, como Colombia, Ecuador y Perú cuentan con medidas proteccionistas como registro previo de importaciones, obligación de contratar una verificadora y presentación detallada de los lotes; en Venezuela los productos confeccionados ingresan sin impedimentos, con sólo cumplir el precio mínimo de referencia. Por lo cual, los fabricantes de ropa requieren de una supervisión más exhaustiva del sector (con el fin de garantizar que la producción sólo se realice formalmente y evitar las falsificaciones), así como la automatización de las aduanas para reducir al mínimo los ilícitos de importación.

\section{Características de la industria de la confección en Venezuela}

La industria de la confección venezolana, se caracteriza, al igual que en el resto de los países en desarrollo, por presentar un alto grado de atomización y de informalidad en su estructura empresarial (Rosales, 1994: 19). Dentro de contormación destacan: microempresas familiares, empresas pequeñas, medianas y grandes, donde predominan las empresas de menor tamaño (pequeñas y medianas industrias, no agrupadas en cámaras y/o asociaciones); cabe destacar también, que muchos confeccionistas operan bajo la denominada economía informal. Esta última caracteristica hace que la confección como actividad tienda a crecer en períodos de crisis económicas, ya que es una vía rápida para obtener ingresos.

Por otro lado, existe una alta concentración geográfica alrededor de la región central, asi por ejemplo para 1986 , aproximadamente un $92 \%$ de los establecimientos están ubicados en la ciudad capital, debido a la cercanía de los grandes mercados de consumo final y de factores productivos (Molina y Alonso, 1991: 11). El sector confección Venezolano es intensivo en mano de obra, generaimente de baja calificación, lo que permite catalogarlo como un importante generador de puestos de trabajo, aunque por lo general éste pague salarios mínimos. Por otra parte, este tipo de industrias no requieren de una gran inversión en tecnología y volúmenes de capital de trabajo, por lo cual se puede afirmar que en este sector no existen grandes barreras de entrada.

Otro de los aspectos a considerar está referido a la dinámica del mercado de la confección, el cual presenta un alto nivel de competencia entre los fabricantes nacionales e internacionales. La rivalidad se manifiesta tanto en la competencia formal como en la competencia desleal, siendo una señal clara del deterioro de los términos de las relaciones competitivas la práctica masiva de ilícitos aduaneros, entre los que destaca el contrabando.

Dentro de la industria de la confección es posible distinguir dos fases del proceso productivo: a) El preensamblado; que implica diseñar el modelo según el gusto de los consumidores o del segmento al cual va dirigida la mercancía; esta fase implica también el preparar mol- 
des de tallas o medidas especificas, trazar sobre la tela y cortar la misma. b) EI ensamblaje que se refiere a la organización de piezas ya cortadas, a la costura y al planchado de las prendas. Los productos que se fabrican en el sector confección suelen diferenciarse por medio del diseño, la calidad, la garantía, el tiempo de entrega, el precio, entre otras variables. Estos aspectos son vitales a la hora de evaluar el nivel de competitividad del sector, según se desprende del estudio de competitividad realizado por Raiza Molina y Osvaldo Alonso en 1991.

Este mismo estudio determinó que la estructura de costos de la industria de la confección es la siguiente: "El costo de la mano de obra asciende al $19 \%$, las materias primas representan un $59 \%$ del total de costos mientras que otros insumos explican el $17 \%$, sólo un $5 \%$ del costo responde a pagos por servicios de capital" (1991: 13). Esto último revela en parte por qué el sector confección venezolano posee un bajo nivel tecnológico, así mismo, evidencia un acceso limitado a información actualizada y además sólo pocas empresas participan activamente en asociaciones industriales $y / o$ foros donde se difunde información tecnológica actualizada y ajustada a las necesidades nacionales.

Son varias las opiniones que tratan de justificar la situación planteada en el párrafo anterior. Así, por una parte algunas empresas plantean la dificultad de conseguir recursos humanos calificados y centros de formación que adiestren en el uso de nuevas tecnologías. Otras se sustentan en la falta de asistencia técnica que oriente la selección adecuada de dichas tecnologias y, por último, que éstas resultan muy costosas y no se justifican dados los volúmenes que producen la mayoría de las empresas (pequeñas y medianas), ya que de utilizarlas, ello sólo redundaría en un aumento de la ya elevada capacidad ociosa de los equipos. En el sector se utiliza la fuerza mecánica para ayudar al trabajador a realizar muchas labores, pero es quizás ésta una de las industrias en las que menos se ha avanzado hacia la mecanización y automatización.

\section{Desempeño reciente del sector confección en la región zuliana}

Tradicionalmente la industria de la confección ha sido por excelencia un sector generador de valor agregado y altamente empleador de mano de obra, sin embargo, en el caso venezolano el impacto de este sector es relativamente pequeño dadas las dimensiones que el mismo tiene en el pais.

Durante el período 1989-1995 la evolución del sector confección puede analizarse al menos en tres sub-periodos, tomando en consideración los datos estadísticos aportados por la encuesta industrial realizada por la OCEI.

\section{De 1989 a 1990: La onda contractiva.}

Este sub-período se caracterizó por notables cambios en la política económica que afectaron de forma importante la evolución del sector confección. Como se recordará, hasta este momento el sector gozaba de una gran protección e 
incentivos gubernamentales; luego con la eliminación de los subsidios, el levantamiento de los controles establecidos en las principales variables macroeconómicas y la apertura de la economía, el gobierno se vio obligado a desconocer las cartas de crédito firmadas por la administración anterior, provocando para 1989 una crisis financiera en el sector, lo cual se tradujo en el cierre de algunas empresas, y la caída de la producción.

Al cierre de 1990 el valor agregado del sector cae en un $22,12 \%$ con respecto al valor alcanzado en 1989 , siendo más acentuada la caída en el sub-sector que agrupa a las grandes industrias al experimentar una disminución equivalente al $28,32 \%$; sin embargo, al mismo tiempo destaca el vertiginoso crecimiento del sub-sector que agrupa a la pequeña industria, el cual se elevó en un $125,31 \%$ en comparación con las cifras alcanzadas en 1989 (Ver Figura 1). Aún cuando no existe una razón evidente que fundamente el mencionado incremento, un argumento que adquiere importancia es el hecho de que la crisis económica origina un aumento del número de desempleados, quienes con el propósito de solucionar su problema de empleo ven la posibilidad de crear una micro o pequeña empresa que genere los ingresos para su subsistencia.

Este comportamiento marcadamente opuesto entre las grandes y pequeñas industrias, refleja el impacto que las políticas de apertura comercial iniciadas a principios de 1989 ejercieron sobre la gran industria; las evidencias demuestran que la influencia de las medidas de ajuste expandió rápidamente su onda contractiva en las empresas de mayor ta- maño y por tanto menos susceptibles a una adaptación rápida.

Por otro lado para 1989 y 1990 la participación de la Gran industria en el valor agregado total osciló entre el $86,55 \%$ y el $79,66 \%$ mientras, que la participación de la pequeña industria sólo alcanzó el $20,34 \%$ en 1990 , cuestión que fundamenta el por qué la caída del sub-sector de la gran industria influyó más en el retroceso del valor agregado del sector confección que el crecimiento de la pequeña industria (Ver Figura 2).

La evolución del número de establecimientos registró un crecimiento del $10 \%$ para el cierre de 1990 (en términos comparativos según cifras del año anterior), esto debido probabiemente a que detener los proyectos de instalación de establecimientos ya en marcha, resultaba más costoso que asumir el riesgo de las nuevas políticas de apertura. Dicho crecimiento estuvo por cierto apalancado por el sub-sector de la pequeña industria, cuestión perfectamente explicable dado el crecimiento del valor agregado en este sub-sector (Ver Figura 3).

Con respecto al personal ocupado en la industria de la confección, se registra una variación negativa equivalente al $6,56 \%$ entre 1989 y 1990 (situación similar al caso del valor agregado), a pesar de que el dinamismo experimentado por la pequeña industria aumentó el empleo, el mismo no fue suficiente para compensar la caída registrada en los sub-sectores de la mediana y gran industria (Ver Figura 4). De hecho, estas empresas tenian para este entonces una participación del $83,84 \%$ frente a una participación del $16,16 \%$ de la pequeña industria (Ver Figura 5). 
Industria de la confección y competitividad

Romero de Cuba, Jenny y otros

\section{Figura 1}

Región Zuliana. Valor agregado de la industria de la confección.

Tasas de Variación. Período 1989-1995

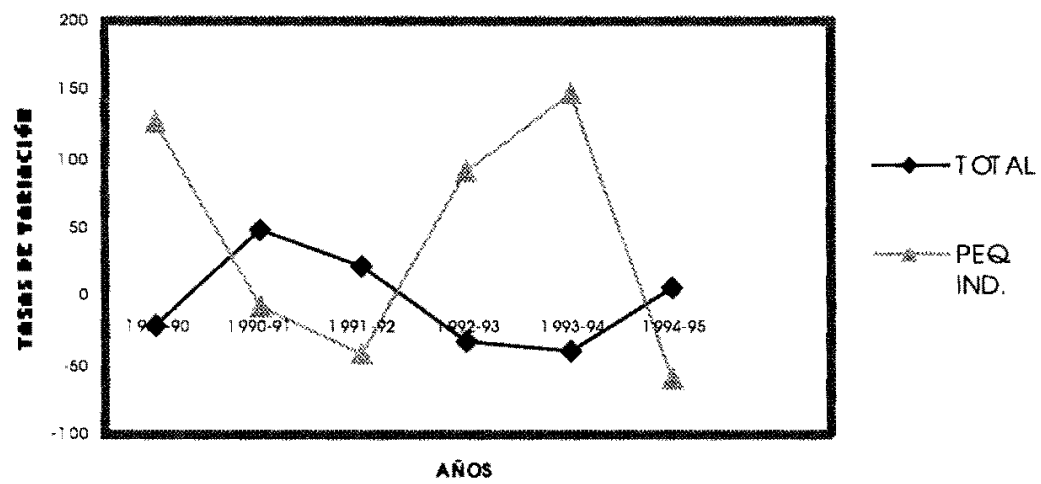

Fuente: Cálculos propios con base en la información de la Encuesta Industrial de la O.C.E.I.

Figura 2

Región Zuliana

Valor agregado de la Industria de la Confección.

Participaciones Porcentuales (\%). Período 1989-1995.

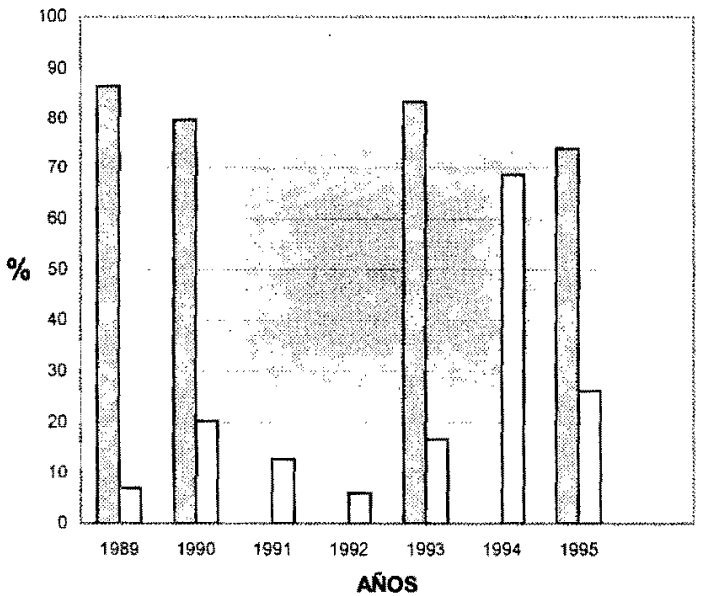

DGRAN IND

DPEO INO

Fuente: Cálculos propios con base en la información de la Encuesta Industrial de la O.C.E.I. Periodo 1989-1995.

Nota: La Gran Industria no dispone de datos para los años: 1991, 1992 y 1994. 
Figura 3

Región Zuliana.

Número de Establecimiento de la Industria de la Confección.

Tasas de Variación. Período 1989-1995

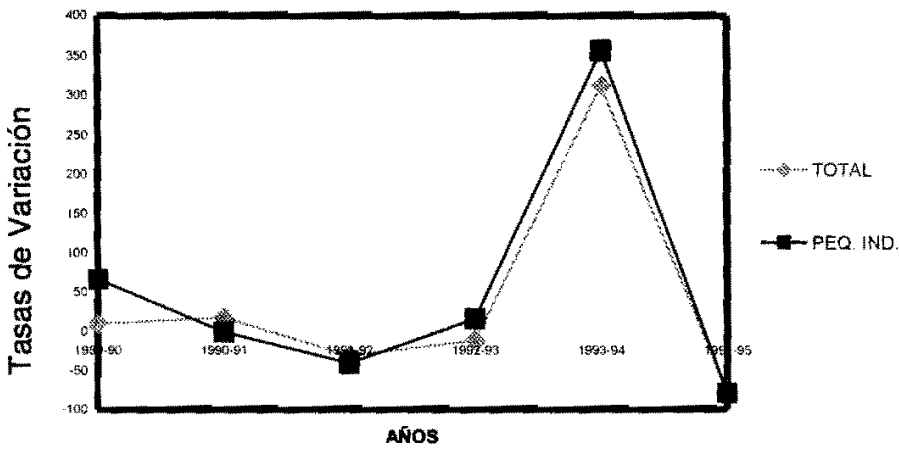

Fuente: Cálculos propios con base en la información de la Encuesta Industrial de la O.C.E.I. 1989-1995.

Nota: La Pequeña Industria no dispone de información para el periodo 1990-91.

Figura 4

Región Zuliana

Personal ocupado en la Industria de la Confección.

Tasas de Variación. Período 1989-1995

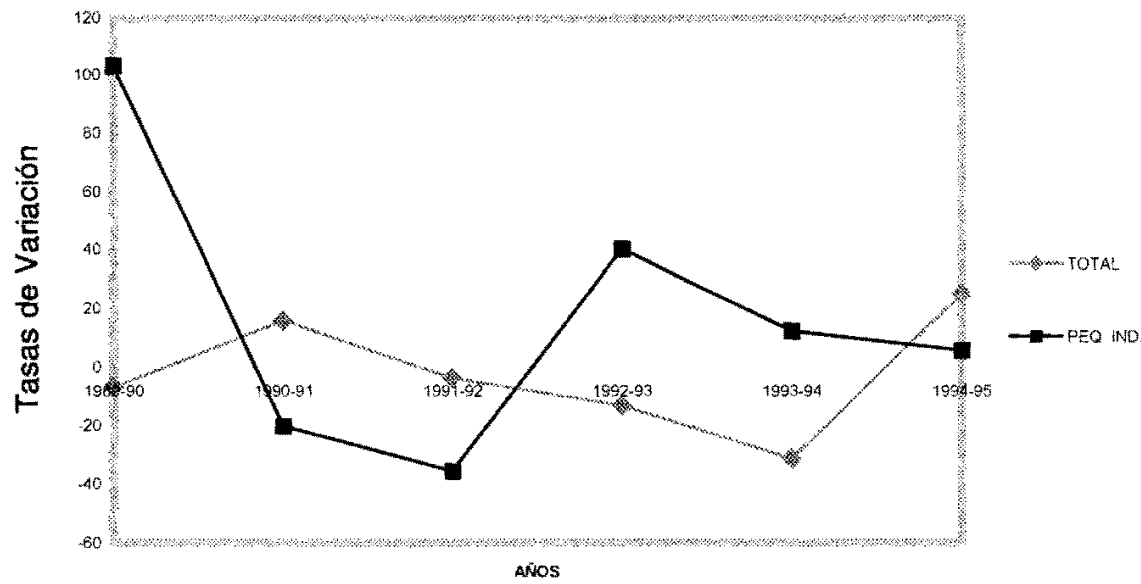

Fuente: Cálculos propios con base en la información de la Encuesta Industrial de la O.C.E.I. 1989-1995. 


\section{Figura 5}

Región Zuliana.

\section{Personal ocupado en la Industria de la Confección.}

Participaciones Porcentuales (\%).

Período 1989-1995.

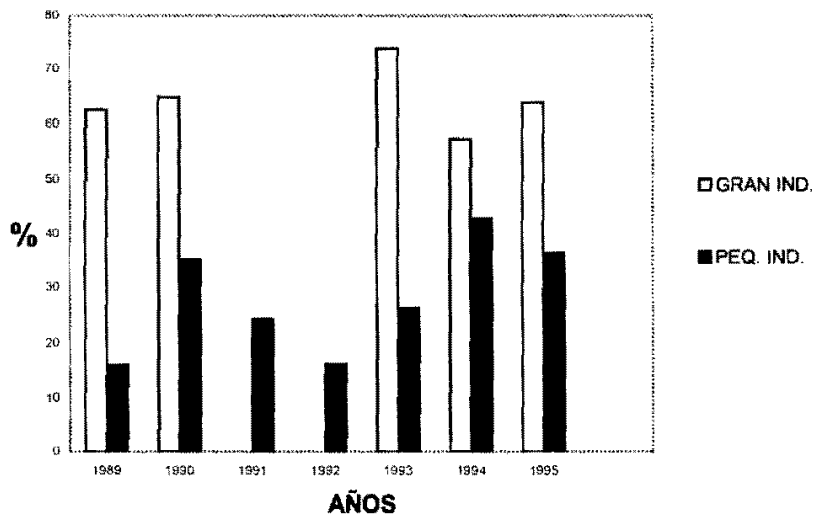

Fuente: Cálculos propios con base en la información de la Encuesta Industrial de la O.C.E.I. Periodo 1989-1995.

Nota: La Gran Industria no disponde de información para los años 1991 y 1992.

Durante este sub-período, además de reducirse el número de personas empleadas en el sector confección, también se produce una reducción de los sueldos y salarios pagados en la industria (Ver Figura 6 ).

\section{De 1991 a 1992: La expansión.}

Una vez superado el impacto contractivo inicial, la industria de la confección entró en la senda del crecimiento, registrando el valor agregado tasas que superaban ampliamente el $20 \%$. Al respecto, en el subperiodo 1990-1991 se evidenció un incremento del $46,85 \%$, recuperando de esta forma la caida experimentada al cierre de 1989; posteriormente, entre los años 1991-1992 disminuye su dinamismo para ubicarse en un
$20,93 \%$. Este comportamiento obedece a la expansión del estrato de la gran industria, dado que la pequeña industria registra un descenso del $8,65 \%$ y $42,88 \%$ respectivamente (Ver Figura 1).

El empleo en el sector confección mostró una evolución positiva para 1990 1991 (16,13\%), sin embargo, la tendencia no se mantiene y al cierre de 1992 la población ocupada en esta industria desciende en un $3,54 \%$ con respecto a 1991 , esto debido probablemente a que las intentonas de golpe de Estado generaron expectativas negativas entre los empresarios del sector, incidiendo en la disminución de la demanda de mano de obra (Ver Figura 4).

La variable "sueldos y salarios" evidenció una situación similar a la del empleo, pues luego de una importante recu- 


\section{Figura 6}

Región Zuliana

Sueldos y Salarios pagados en la Industria de la Confección.

Tasas de Variación. Período 1989-1995.

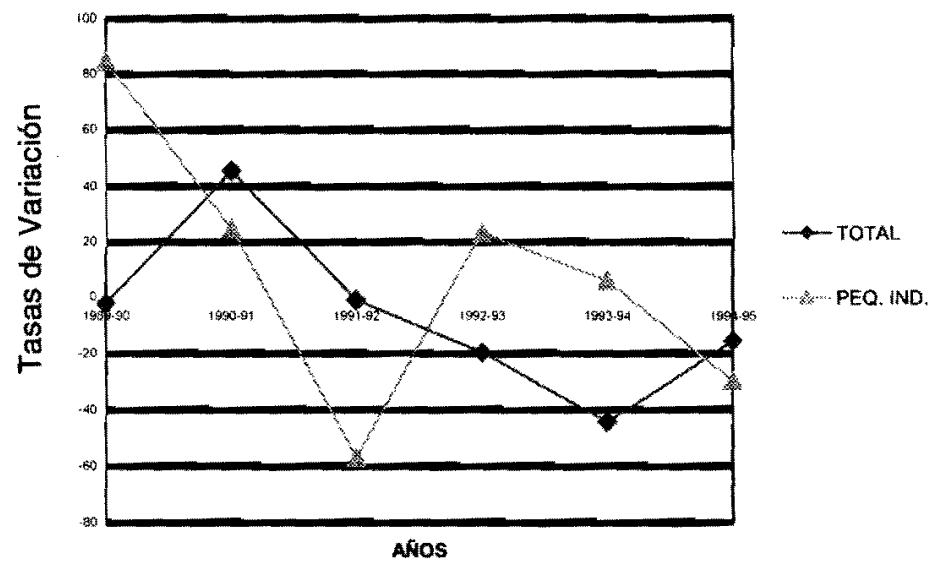

Fuente: Cálculos propios con base en la información de la Encuesta Industrial de la O.C.E.I.

Período 1989-1995.

peración de los sueldos pagados en 1991 $(45,6 \%)$, siguió una disminución relativamente pequeña al cierre de 1992 equivalente al 1.01\% (Ver Figura 6).

Siguiendo la misma tendencia mostrada por las variables anteriores, el número de establecimientos en la industria de la confección creció a una tasa del $18,18 \%$ en 1991 , pero este incremento se detuvo al final de 1992, al caer en un $30,77 \%$, ésta caída obedece entre otras causas al enrarecido clima político reinante para el año 1992 (Ver Figura 3).

\section{De 1993 a 1995: Del Gran Viraje al Barco sin Rumbo.}

La inestabilidad política y social imperante durante este período, así como la falta de direccionalidad de la política eco- nómica, produjeron una dramática caída del valor agregado de la industria de la confección, la cual se ubicó en un $31,72 \%$ al cierre de 1993, mientras que para 1994 alcanzó un $40,20 \%$. La tendencia contractiva en esta variable cambia para 1995, donde se evidenció una leve recuperación del $4,75 \%$, lo cual es favorable a pesar de los controles de cambio existentes así como de la reactivación de acuerdos con paises latinoamericanos (Pacto Andino, Grupo de los tres, Grupo de río, etc.), que develaron el bajo nivel competitivo de la industria de la confección venezolana frente a países como Colombia, Brasil, México, entre otros. Durante todo este período sólo el sub-sector de la pequeña industria registró un crecimiento importante equivalente al $90,2 \%$ al final de 1993 y $146,34 \%$ en 1994 , demostran- 
do una vez más su evolución en sentido opuesto al de la mediana y gran empresa (Ver Figura 1).

Si los resultados de la evolución del valor agregado evidenciaron los efectos de la crisis política y económica del pais, con mayor razón aún, ésta se dejó sentir en el caso del empleo, e incluso anticipándose ya desde el cierre de 1992, de esta forma el personal ocupado en la industria de la confección desciende sucesivamente a tasas del $13,09 \%$ en 1993 y $31,02 \%$ en 1994. Para 1995 se produjo un aumento en el empleo equivalente al $25,33 \%$ con respecto a 1994 , hecho explicable en vista de la recuperación del valor agregado (Ver Figura 4). Así mismo, los salarios pagados en este sector caen a tasas del $19,47 \%, 44,44 \%$ y $15,45 \%$ durante el lapso $1993-1995$ (Ver Figura 6).
Para el cierre de 1993 el número de establecimientos registró una variación negativa, al caer a una tasa del $11,11 \%$ con respecto a 1992, lo cual es coherente con la evolución del valor agregado, sin embargo, al cierre de 1994 el número de establecimientos evidencia un crecimiento impactante con respecto al año anterior, al registrar una tasa de variación positiva del $312,5 \%$ (Ver Figura 3 ), la cual estuvo fundamentada en la participación de la pequeña industria que alcanzó el $96,97 \%$ del total de establecimientos, mientras que la gran industria concentró sólo el 3,03\% de los mismos (Ver Figura 7). Tal comportamiento podría deberse a que con la acentuación de la crisis económica se generó una desocupación de recursos (capital, mano de obra, entre otros), que probablemente se desplazaron hacia el sector confección, dado que

\section{Figura 7}

Región Zuliana

Número de establecimientos en la industria de la Confección. Participaciones porcentuales (\%). Período 1989-1995.

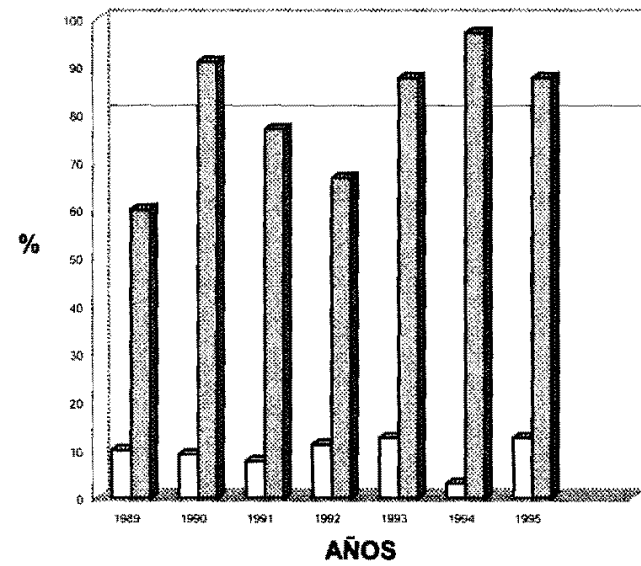

DGRAN IND.

QPEQ. IND.

Fuente: Cálculos propios con base en la información de la Encuesta Industrial de la O.C.E.I.

Período 1989-1995. 
el mismo requiere bajos niveles de capital y no presenta grandes barreras de entrada esto, aunado al aumento de expectativas positivas generadas por el inicio del nuevo gobierno explican esta situación. Al cierre de 1995, los efectos de la crisis financiera iniciada en 1994 se dejaron sentir en el sector confección, traduciéndose en un cambio negativo con respecto a los resultados obtenidos en el año anterior, registrando una disminución equivalente al $75,75 \%$, dado que el aumento de las tasas activas se tradujo en un enorme costo financiero, difícil de enfrentar, fundamentalmente para los pequeños y medianos industriales.

\section{Conclusiones}

Indudablemente las políticas de ajuste macroeconómico y en particular las políticas de apertura comercial impactaron contractivamente al sector confección, revelando la poca capacidad de estas industrias para enfrentar la competencia externa, tanto en el plano nacional como internacional. Este resultado obedece a una visión empresarial restringida de las formas de competencia, fundamentada en el apoyo del Estado, dejando de lado el reto de la competitividad internacional. Sin embargo, en la actualidad es inevitable la incorporación al proceso de globalización de mercados lo que necesariamente exige un cambio en la visión de negocios de los empresarios venezolanos.

El estudio de las variables consideradas durante el período $1989-1995$, refleja un comportamiento irregular del sector, evidenciado en aumentos y disminuciones de proporciones considerables, especialmente en el caso del valor agregado y el personal ocupado. No obstante, el proceso de ajustes demostró el alto nivel de flexibilidad de la pequeña industria para adaptarse a los cambios del entorno competitivo, producto de los bajos requerimientos de capital y de la relativa inexistencia de barreras de entradas al sector.

A pesar del desempeño negativo evidenciado por la industria a lo largo del periodo, es posible pensar en la posibilidad de aprovechar la ventaja que brinda el bajo costo de la mano de obra, pues el pais ha venido atravesando en los últimos años por un proceso de franco deterioro salarial, que ha llevado a ubicarlo para 1997 entre los seis paises de América Latina y el Caribe con salario mínimo real unano más bajo, aspecto este que ha sido identificado como una las ventajas competitivas estáticas fundamentales para desarrollar la cadena textil-confección en algunas economias asiáticas. Así mismo, el éxito en la inclusión del mercado de textiles y prendas de vestir dependerá de la capacidad para modernizar las estructuras productivas de sus industrias, esto para poder enfrentar los cambios que se avizoran en el comercio internacional, con la expedición de los acuerdos sobre acceso a mercados, por ejemplo, con el acuerdo de multifibras que expedirán en el año 2005, a partir de ese momento se libera el comercio de textiles.

En este sentido, los países en desarrollo deben competir entre sí para conquistar nuevos mercados. La competencia no sólo debe consistir en aprovechar las ventajas comparativas naturales o estáticas como materia prima o mano de obra barata, sino también en potenciar las ventajas competitivas dinámicas, 
como la velocidad de respuesta a los cambios de la moda, los tiempos de entrega y el logro de acuerdos políticos y preferenciales que garanticen una inserción favorable en el comercio mundial.

\section{Bibliografía citada}

Bitar, Sergio y Troncoso, Eduardo (1983). EI Desafio Industrial de Venezuela. Editorial Pomaire. Caracas.

Comisión Económica para América Latina y el Caribe (CEPAL) (1997). Estudio Económico para América Latina y el Caribe.

Chiappe, Giuliana (1999). Cerraron 320 Industrias Textiles. Diario El Universal. Cuerpo 2. Caracas.

Corona Guzmán, Roberto (1996). El entorno económico internacional en un mundo más interdependiente. Revista Comercio Exterior. Banco Nacional de Comercio Exterior, S.N.C. Vol. 46, Núm. 11. México. Págs. 911-921.

Kouzmine, Valentine (1997). Acceso de los Productos de América Latina y el Caribe al Mercado de Textiles y Vestido de los Estados Unidos. Documento de las Naciones Unidas. Co- misión Económica para América Latina y el Caribe (CEPAL).

Maza Zavala, D.F. (1999). Desarrollo Sustentable, Equidad, Competitividad y Globalización. Revista Desarrollo Indoamericano. No. 106. Año XXXIII. Universidad Simón Bolivar. Corporación Simón Bolívar. Barranquilla-Colombia. Págs. 11-16.

Molina, Raiza y Alonso, Osvaldo (1991). Estudio de Competitividad del Sector Confección en Venezuela. Instituto Latinoamericano de Investigaciones Sociales (ILDIS) y otros. Caracas.

Oficina Central de Estadistica e Informática (OCEI) (1989). 25 años Encuesta Industrial. Caracas.

Oficina Central de Estadística e Informática (OCEI). Encuesta Industrial-Región Zuliana. Periodo 1989-1995.

Rosales, Ramón (1994). Industria Textil. Documento de base Proyecto Venezuela Competitiva. No. 8. Ediciones IESA. Caracas.

Toledano, Andeab y Castillo, Manuel (1997). Estrategias disponibles para mejorar la competitividad de manufactura en el sector confección de Venezuela. IESA. Trabajo de Grado. 